\title{
Evaluation of CSF flow in shunts using a non-invasive thermal technique
}

\author{
Gani Abazi*1, Laurel Fleming ${ }^{1}$, Marek Swoboda ${ }^{2}$, Tomer Anor ${ }^{1}$ and \\ Joseph R Madsen ${ }^{1}$
}

\author{
Address: ${ }^{1}$ Department of Neurosurgery, Children's Hospital Boston, Harvard Medical School, 300 Longwood Avenue, Boston, MA 02115, USA \\ and ${ }^{2}$ Neuro Diagnostic Devices, 3701 Market St. Fourth Floor, Philadelphia, PA 19104, USA \\ Email: Gani Abazi* - gani.abazi@childrens.harvard.edu \\ * Corresponding author
}

from 52nd Annual Meeting of the Society for Research into Hydrocephalus and Spina Bifida

Providence, RI, USA. II-I4 June 2008

Published: 3 February 2009

Cerebrospinal Fluid Research 2009, 6(Suppl I):S4I doi:I0.II86/I743-8454-6-SI-S4I

This abstract is available from: http://www.cerebrospinalfluidresearch.com/content/6/SI/S4 I

(C) 2009 Abazi et al; licensee BioMed Central Ltd.

\begin{abstract}
Background
Cerebrospinal fluid (CSF) shunts have dramatically improved effective treatment for patients with hydrocephalus, but fundamental aspects of their function, including typical rates of fluid flow through the system, remain unknown. Despite advances in the quality of shunts, shunt failure rates remain significantly high and evaluation of CSF flow in shunts with invasive techniques continues to present diagnostic and clinical difficulties involving patients' comfort and hospital resources.
\end{abstract}

Shuntcheck is an FDA approved non-invasive diagnostic device for detecting the presence of CSF flow in the subcutaneous tubing of hydrocephalic patients. Shuntcheck uses thermal sensors to detect convection differences in shunts with flowing CSF and the surrounding subcutaneous tissue. The potential role of this technology in the clinical decision-making process remains to be defined.

\section{Materials and methods}

Twenty-six shunted hydrocephalic patients aged five months to 27 years with subcutaneous ventriculo peritoneal (VP) shunts without signs and symptoms of shunt malfunction were tested during their routine follow up visits in a neurosurgical clinic. The Shuntcheck test involves putting three sensors - proximal, distal and control - over the skin, in line with the subcutaneous shunt and placing an ice cube over the shunt proximally to sen- sors for 60 seconds. The Shuntcheck device starts recording 10 seconds before the ice cube application and continues recording during the cooling period and after ice removal for eight minutes. Cutaneous thermal differences are displayed graphically, indicating if there is CSF flow in the shunt. The test was done in three positions, with the patient lying supine, at 45 degree angle and sitting upright.

\section{Results \\ CSF flow was detected in 24 out of 26 patients or $92 \%$ of the tests. However, a significant variability in regard with flow detection has been detected between testing posi- tions, with $58 \%$ of the patients showing flow in the supine position, and $70 \%$ showing flow at 45 degree and sitting positions. The proximal sensor typically shows the highest amplitude of temperature effect. Results are comparable with previous Shuntcheck studies done in animals.}

\section{Conclusion}

Detection of flow is maximized with Shuntcheck thermal technique by using at least two different testing positions. Special care should be given to patients under three years of age as a result of their movement during the test and interference this may cause with recordings. Shuntcheck test in sitting position produces the highest flow detection rate and has been evaluated to produce the least patient discomfort 